\title{
On directional derivative sets of the max-min set-valued maps
}

\author{
Erdal Ekici
}




\title{
ON DIRECTIONAL DERIVATIVE SETS OF MAX-MIN SET-VALUED MAPS
}

\author{
ERDAL EKICI
}

Received 2 February, 2005

\begin{abstract}
In this paper, necessary statements are given for a minimum point and a maximum point of the max-min function. Moreover estimations for the directional lower and upper derivative sets of the max-min set-valued map which are used to state a characterization of the directional derivative of the max-min functions are given. Furthermore, a sufficient condition ensuring the existence of the directional derivative of the max-min function is obtained by using the lower differentiability of the max-min set-valued maps.
\end{abstract}

1991 Mathematics Subject Classification: 26E25

Keywords: set-valued map, derivative set, max-min function

\section{INTRODUCTION}

The directional derivatives of max-min functions were studied in [7]. It is wellknown that max-min functions are considered and occur in control theory problems, parametric optimization problems and differential game theory problems [4]. Moreover marginal functions are max-min functions (see [3-6, 10-14]).

In this paper, necessary statements are given for a minimum point and a maximum point of the max-min function. It is well-known that the directional lower and upper derivative sets of max-min set valued maps are used to state a characterization of the directional derivative of max-min function [7]. In this paper, estimations for the directional lower and upper derivative sets of max-min set valued maps are given. Furthermore, a sufficient condition ensuring the existence of the directional derivative of max-min function is obtained by using the lower differentiability of max-min setvalued maps.

In this study, $\operatorname{cl}\left(\mathbb{R}^{m}\right)\left(\operatorname{comp}\left(\mathbb{R}^{m}\right)\right)$ denotes the set of all nonempty closed (compact) subsets in $\mathbb{R}^{m}$. Let $a(\cdot): \mathbb{R}^{n} \rightarrow \operatorname{cl}\left(\mathbb{R}^{m}\right)$ be an upper semi-continuous set-valued map. For $(x, y) \in \mathbb{R}^{n} \times \mathbb{R}^{m}$ and vector $f \in \mathbb{R}^{n}$, let us consider the following sets:

$$
\begin{gathered}
D a(x, y) \mid(f)=\left\{d \in \mathbb{R}^{m}: \liminf _{\delta \rightarrow+0} \delta^{-1} \operatorname{dist}(y+\delta d, a(x+\delta f))=0\right\}, \\
D^{*} a(x, y) \mid(f)=\left\{v \in \mathbb{R}^{m}: \lim _{\delta \rightarrow+0} \delta^{-1} \operatorname{dist}(y+\delta d, a(x+\delta f))=0\right\},
\end{gathered}
$$


where $x \in \mathbb{R}^{n}, D \subset \mathbb{R}^{n}, \operatorname{dist}(x, D)=\inf _{d \in D}\|x-d\| . D a(x, y) \mid(f)\left(D^{*} a(x, y) \mid\right.$ $(f))$ is called the upper (lower) derivative set of the set-valued map $a(\cdot)$ at $(x, y)$ in direction $f$. Note that the directional upper (lower) derivative set of the set-valued map $a(\cdot)$ is closed and there is a connection between the upper (lower) derivative set of a set-valued map and the upper (lower) contingent cone which is used to investigate several problems in nonsmooth analysis [1,2,8]. It is obvious that $D^{*} a(x, y) \mid(f) \subset$ $\operatorname{Da}(x, y) \mid(f)$. The symbol

$$
A=\operatorname{gr} a(\cdot)=\left\{(x, y) \in \mathbb{R}^{n} \times \mathbb{R}^{m}: y \in a(x)\right\}
$$

denotes the graph of the set-valued map $a(\cdot)$. Since $a(\cdot)$ is upper semicontinuous, $A$ is a closed set. It is possible to show that $D a(x, y)\left|(f)=D^{*} a(x, y)\right|(f)=\varnothing$ if $(x, y) \notin A, D a(x, y)\left|(f)=D^{*} a(x, y)\right|(f)=\mathbb{R}^{m}$ if $(x, y) \in \operatorname{int} A$, where int $A$ denotes the interior of $A$.

Let $f(\cdot): \mathbb{R}^{n} \rightarrow \mathbb{R}$ be a function. The lower and upper derivative of $f(\cdot)$ at the point $x$ in direction $v$ are denoted by the symbols $\frac{\partial^{-} f(x)}{\partial v}$ and $\frac{\partial^{+} f(x)}{\partial v}$ respectively, and defined by the formulas

$$
\frac{\partial^{-} f(x)}{\partial v}=\liminf _{\delta \rightarrow+0}[f(x+\delta v)-f(x)] \delta^{-1},
$$

and

$$
\frac{\partial^{+} f(x)}{\partial v}=\limsup _{\delta \rightarrow+0}[f(x+\delta v)-f(x)] \delta^{-1} .
$$

If

$$
\frac{\partial f(x)}{\partial v}=\lim _{\delta \rightarrow+0}[f(x+\delta v)-f(x)] \delta^{-1}
$$

exists and is finite, then $f(\cdot)$ is said to be differentiable at the point $x$ in direction $v$ and $\frac{\partial f(x)}{\partial v}$ denotes the derivative of $f(\cdot)$ at the point $x$ in direction $v$.

Let $a(\cdot): \mathbb{R}^{n} \rightarrow \operatorname{comp}\left(\mathbb{R}^{m}\right), b(\cdot): \mathbb{R}^{n} \rightarrow \operatorname{comp}\left(\mathbb{R}^{k}\right)$ be set-valued maps and $\sigma(\cdot, \cdot, \cdot)$ : $\mathbb{R}^{n} \times \mathbb{R}^{m} \times \mathbb{R}^{k} \rightarrow \mathbb{R}$ be a continuous function on $\mathbb{R}^{n} \times \mathbb{R}^{m} \times \mathbb{R}^{k}$. The max-min function is denoted by $m(\cdot)$ and is defined by

$$
m(x)=\max _{y \in a(x)} \min _{z \in b(x)} \sigma(x, y, z) .
$$

In this paper, we will assume that $a(\cdot): \mathbb{R}^{n} \rightarrow \operatorname{comp}\left(\mathbb{R}^{m}\right), b(\cdot): \mathbb{R}^{n} \rightarrow \operatorname{comp}\left(\mathbb{R}^{k}\right)$ are continuous set-valued maps and $\sigma(\cdot, \cdot, \cdot): \mathbb{R}^{n} \times \mathbb{R}^{m} \times \mathbb{R}^{k} \rightarrow \mathbb{R}$ is a continuous function on $\mathbb{R}^{n} \times \mathbb{R}^{m} \times \mathbb{R}^{k}$ and locally Lipschitz on $\mathbb{R}^{m} \times \mathbb{R}^{k}$, i. e., for every bounded $D \subset \mathbb{R}^{n} \times \mathbb{R}^{m} \times \mathbb{R}^{k}$, there exists $L(D)>0$ such that

$$
\left|\sigma\left(x, y_{1}, z_{1}\right)-\sigma\left(x, y_{2}, z_{2}\right)\right| \leq L(D)\left\|\left(y_{1}-y_{2}, z_{1}-z_{2}\right)\right\|
$$

for any $\left(x, y_{1}, z_{1}\right),\left(x, y_{2}, z_{2}\right) \in D$. Under these conditions $m(\cdot)$ is a continuous function (see [1]). Let 


$$
\begin{aligned}
Y_{*}(x)=\left\{\left(y_{*}, z_{*}\right) \in a(x) \times b(x):\right. & \\
& \left.m(x)=\max _{y \in a(x)} \min _{z \in b(x)} \sigma(x, y, z)=\sigma\left(x, y_{*}, z_{*}\right)\right\} .
\end{aligned}
$$

The map $x \mapsto Y_{*}(x)$ is an upper semicontinuous set-valued map and it is called a max-min set-valued map.

\section{Minimization AND MAXIMIZATION PROBLEMS OF MAX-MIN FUNCTION}

Now we give necessary statements for a minimum point and a maximum point of max-min function.

Theorem 1. Let the function $m(\cdot): \mathbb{R}^{n} \rightarrow \mathbb{R}$ be in the form (1.1). Suppose that $x_{*} \in \mathbb{R}^{n}$ is a minimum point of max-min function $m(\cdot)$. Then

$$
\inf _{f \in \mathbb{R}^{n}} \inf _{(y, z) \in Y_{*}\left(x_{*}\right)} \inf _{(d, n) \in D Y_{*}\left(x_{*}, y, z\right) \mid(f)} \frac{\partial^{+} \sigma\left(x_{*}, y, z\right)}{\partial(f, d, n)} \geq 0 .
$$

Proof. Let $f \in \mathbb{R}^{n}$. Since $x_{*} \in \mathbb{R}^{n}$ is a minimum point of max-min function $m(\cdot)$, then it follows that $m\left(x_{*}+\delta f\right) \geq m\left(x_{*}\right)$ for all $\delta>0$. In that case, it follows from here that

$$
\frac{\partial^{-} m\left(x_{*}\right)}{\partial f}=\liminf _{\delta \rightarrow+0} \frac{1}{\delta}\left[m\left(x_{*}+\delta f\right)-m\left(x_{*}\right)\right] \geq 0 .
$$

Hence, from here and of [7, Proposition 7], we have

$$
0 \leq \frac{\partial^{-} m\left(x_{*}\right)}{\partial f} \leq \inf _{(y, z) \in Y_{*}\left(x_{*}\right)} \inf _{(d, n) \in D Y_{*}\left(x_{*}, y, z\right) \mid(f)} \frac{\partial^{+} \sigma\left(x_{*}, y, z\right)}{\partial(f, d, n)}
$$

and hence we obtain the inequality.

Theorem 2. Let the function $m(\cdot): \mathbb{R}^{n} \rightarrow \mathbb{R}$ be in the form (1.1). Suppose that $x_{*} \in \mathbb{R}^{n}$ is a maximum point of max-min function $m(\cdot)$ and there exists a $(y, z) \in$ $Y_{*}\left(x_{*}\right)$ such that $D Y_{*}\left(x_{*}, y, z\right) \mid(f) \neq \varnothing$ for all $f \in \mathbb{R}^{n}$. Then

$$
\sup _{f \in \mathbb{R}^{n}} \inf _{(y, z) \in Y_{*}\left(x_{*}\right)} \inf _{(d, n) \in D Y_{*}\left(x_{*}, y, z\right) \mid(f)} \frac{\partial^{-} \sigma\left(x_{*}, y, z\right)}{\partial(f, d, n)} \leq 0 .
$$

Proof. Let $f \in \mathbb{R}^{n}$. Since $x_{*} \in \mathbb{R}^{n}$ is a maximum point of max-min function $m(\cdot)$, then it follows that $m\left(x_{*}+\delta f\right) \leq m\left(x_{*}\right)$ for all $\delta>0$. In that case, it follows from here that

$$
\frac{\partial^{+} m\left(x_{*}\right)}{\partial f}=\limsup _{\delta \rightarrow+0} \frac{1}{\delta}\left[m\left(x_{*}+\delta f\right)-m\left(x_{*}\right)\right] \leq 0 .
$$

Hence, from here and [7, Proposition 8], we have

$$
0 \geq \frac{\partial^{+} m\left(x_{*}\right)}{\partial f} \geq \inf _{(y, z) \in Y_{*}\left(x_{*}\right)} \inf _{(d, n) \in D Y_{*}\left(x_{*}, y, z\right) \mid(f)} \frac{\partial^{-} \sigma\left(x_{*}, y, z\right)}{\partial(f, d, n)}
$$

and hence we obtain the inequality. 


\section{DiRECTIONAL DERIVATIVE SETS OF MAX-MIN SET-VALUED MAPS}

Now we give the estimations for the directional lower and upper derivative sets of max-min set-valued map which are used to state a characterization of the directional derivative of the max-min functions in [7].

Let us take the max-min function $m(\cdot)$ such as:

$$
m(x)=\min _{y \in a(x)} \sigma(x, y)
$$

where $a(\cdot): \mathbb{R}^{n} \rightarrow \operatorname{comp}\left(\mathbb{R}^{m}\right)$ is a continuous set-valued map and $\sigma(\cdot, \cdot): \mathbb{R}^{n} \times \mathbb{R}^{m} \rightarrow$ $\mathbb{R}$ is a continuous function on $\mathbb{R}^{n} \times \mathbb{R}^{m}$ and locally Lipschitz on $\mathbb{R}^{m}$, i. e., for every bounded $D \subset \mathbb{R}^{n} \times \mathbb{R}^{m}$, there exists $L(D)>0$ such that

$$
\left|\sigma\left(x, y_{1}\right)-\sigma\left(x, y_{2}\right)\right| \leq L(D)\left\|y_{1}-y_{2}\right\|
$$

for any $\left(x, y_{1}\right),\left(x, y_{2}\right) \in D$. Under these conditions $m(\cdot)$ is a continuous function (see [1]). Then we take

$$
Y_{*}(x)=\left\{y_{*} \in a(x): m(x)=\min _{y \in a(x)} \sigma(x, y)=\sigma\left(x, y_{*}\right)\right\} .
$$

Theorem 3. Let the set-valued map $Y_{*}(\cdot): \mathbb{R}^{n} \rightarrow \operatorname{comp}\left(\mathbb{R}^{m}\right)$ be in the form (3.2). Suppose that there exists a $y_{*} \in Y_{*}\left(x_{*}\right)$ such that $\sigma(\cdot, \cdot)$ is a derivable function at the point $\left(x_{*}, y_{*}\right)$ in direction $(f, d)$ for all $d \in \mathbb{R}^{m}$. Then

$$
D^{*} Y_{*}\left(x_{*}, y_{*}\right) \mid(f) \subset\left\{p \in \mathbb{R}^{m}: \inf _{d \in D a\left(x_{*}, y_{*}\right) \mid(f)} \frac{\partial \sigma\left(x_{*}, y_{*}\right)}{\partial(f, d)}=\frac{\partial \sigma\left(x_{*}, y_{*}\right)}{\partial(f, p)}\right\} .
$$

Proof. Let $y_{*} \in Y_{*}\left(x_{*}\right)$ such that $\sigma(\cdot, \cdot)$ is a derivable function at the point $\left(x_{*}, y_{*}\right)$ in direction $(f, d)$ for all $d \in \mathbb{R}^{m}$.

Let $D^{*} Y_{*}\left(x_{*}, y_{*}\right) \mid(f)=\varnothing$. Then the statement (3.3) holds.

Let $\operatorname{Da}\left(x_{*}, y_{*}\right) \mid(f)=\varnothing$. Then

$$
\inf _{d \in D a\left(x_{*}, y_{*}\right) \mid(f)} \frac{\partial \sigma\left(x_{*}, y_{*}\right)}{\partial(f, d)}=+\infty .
$$

Since $D^{*} Y_{*}\left(x_{*}, y_{*}\right)\left|(f) \subset D^{*} a\left(x_{*}, y_{*}\right)\right|(f) \subset D a\left(x_{*}, y_{*}\right) \mid(f)$, then it follows that statement (3.3) holds.

Now $D^{*} Y_{*}\left(x_{*}, y_{*}\right) \mid(f) \neq \varnothing$. Take $p \in D^{*} Y_{*}\left(x_{*}, y_{*}\right) \mid(f)$. Then from the definition of $D^{*} Y_{*}\left(x_{*}, y_{*}\right) \mid(f)$, there exists a $\delta_{*}>0$ such that for all $\delta \in\left[0, \delta_{*}\right]$,

$$
y_{*}(\delta)=y_{*}+\delta p+o_{1}(\delta) \in Y_{*}\left(x_{*}+\delta f\right)
$$

where $\left\|o_{1}(\delta)\right\| \delta^{-1} \rightarrow 0$ as $\delta \rightarrow+0$. Since

$$
Y_{*}(x)=\left\{y_{*} \in a(x): \sigma(x, y) \geq \sigma\left(x, y_{*}\right), \forall y \in a(x)\right\},
$$

then it follows that for any $y \in a\left(x_{*}+\delta f\right)$,

$$
\sigma\left(x_{*}+\delta f, y\right) \geq \sigma\left(x_{*}+\delta f, y_{*}+\delta p+o_{1}(\delta)\right) .
$$


Choose any $d \in D a\left(x_{*}, y_{*}\right) \mid(f)$. Then from the definition of $D a\left(x_{*}, y_{*}\right) \mid(f)$, there exists a sequence $y_{k} \in a\left(x_{*}+\delta_{k} f\right)$ where $\delta_{k}>0$ and $\delta_{k} \rightarrow+0$ as $k \rightarrow \infty$, such that

$$
y_{k}=y_{*}+\delta_{k} d+o_{2}\left(\delta_{k}\right)
$$

where $\left\|o_{2}\left(\delta_{k}\right)\right\| \delta_{k}^{-1} \rightarrow 0$ as $k \rightarrow \infty$. In that case from (3.4) since $\delta_{k} \rightarrow+0$, then it follows that there exists a $k_{0} \in \mathbb{N}$ such that $\delta_{k} \in\left[0, \delta_{*}\right]$ for all $k \geq k_{0}$ and

$$
\begin{aligned}
\sigma\left(x_{*}+\delta_{k} f, y_{*}+\delta_{k} d+o_{2}\left(\delta_{k}\right)\right) & -\sigma\left(x_{*}, y_{*}\right) \\
& \geq \sigma\left(x_{*}+\delta_{k} f, y_{*}+\delta_{k} p+o_{1}\left(\delta_{k}\right)\right)-\sigma\left(x_{*}, y_{*}\right) .
\end{aligned}
$$

Since the function $\sigma(\cdot, \cdot)$ is locally Lipschitzian on $\mathbb{R}^{m}$, then it follows that for $k=1$, $2, \ldots$, there exists $L_{1}>0$ and $L_{2}>0$ such that

$$
\begin{aligned}
& \left|\sigma\left(x_{*}+\delta_{k} f, y_{*}+\delta_{k} p+o_{1}\left(\delta_{k}\right)\right)-\sigma\left(x_{*}+\delta_{k} f, y_{*}+\delta_{k} p\right)\right| \leq L_{1}\left\|o_{1}\left(\delta_{k}\right)\right\|, \\
& \left|\sigma\left(x_{*}+\delta_{k} f, y_{*}+\delta_{k} d+o_{2}\left(\delta_{k}\right)\right)-\sigma\left(x_{*}+\delta_{k} f, y_{*}+\delta_{k} d\right)\right| \leq L_{2}\left\|o_{2}\left(\delta_{k}\right)\right\| .
\end{aligned}
$$

Consequently, we obtain

$$
\begin{aligned}
& \frac{\partial \sigma\left(x_{*}, y_{*}\right)}{\partial(f, p)=} \lim _{\delta \rightarrow+0}\left[\sigma\left(x_{*}+\delta f, y_{*}+\delta p\right)-\sigma\left(x_{*}, y_{*}\right)\right] \delta^{-1} \\
&= \lim _{k \rightarrow \infty}\left[\sigma\left(x_{*}+\delta_{k} f, y_{*}+\delta_{k} p\right)-\sigma\left(x_{*}, y_{*}\right)\right] \delta_{k}^{-1} \\
&= \lim _{k \rightarrow \infty}\left[\sigma\left(x_{*}+\delta_{k} f, y_{*}+\delta_{k} p\right)-\sigma\left(x_{*}+\delta_{k} f, y_{*}+\delta_{k} p+o_{1}\left(\delta_{k}\right)\right)\right. \\
&\left.\quad+\sigma\left(x_{*}+\delta_{k} f, y_{*}+\delta_{k} p+o_{1}\left(\delta_{k}\right)\right)-\sigma\left(x_{*}, y_{*}\right)\right] \delta_{k}^{-1} \\
& \leq \lim _{k \rightarrow \infty}\left[L_{1}\left\|o_{1}\left(\delta_{k}\right)\right\|+\sigma\left(x_{*}+\delta_{k} f, y_{*}+\delta_{k} p+o_{1}\left(\delta_{k}\right)\right)-\sigma\left(x_{*}, y_{*}\right)\right] \delta_{k}^{-1} \\
&= \lim _{k \rightarrow \infty}\left[\sigma\left(x_{*}+\delta_{k} f, y_{*}+\delta_{k} p+o_{1}\left(\delta_{k}\right)\right)-\sigma\left(x_{*}, y_{*}\right)\right] \delta_{k}^{-1} \\
& \leq \lim _{k \rightarrow \infty}\left[\sigma\left(x_{*}+\delta_{k} f, y_{*}+\delta_{k} d+o_{2}\left(\delta_{k}\right)\right)-\sigma\left(x_{*}, y_{*}\right)\right] \delta_{k}^{-1} \\
&= \lim _{k \rightarrow \infty}\left[\sigma\left(x_{*}+\delta_{k} f, y_{*}+\delta_{k} d+o_{2}\left(\delta_{k}\right)\right)-\sigma\left(x_{*}+\delta_{k} f, y_{*}+\delta_{k} d\right)\right. \\
&\left.+\sigma\left(x_{*}+\delta_{k} f, y_{*}+\delta_{k} d\right)-\sigma\left(x_{*}, y_{*}\right)\right] \delta_{k}^{-1} \\
& \leq \lim _{k \rightarrow \infty} L_{2}\left\|o_{2}\left(\delta_{k}\right)\right\| \delta_{k}^{-1}+\lim _{k \rightarrow \infty}\left[\sigma\left(x_{*}+\delta_{k} f, y_{*}+\delta_{k} d\right)-\sigma\left(x_{*}, y_{*}\right)\right] \delta_{k}^{-1} \\
&= \lim _{k \rightarrow \infty}\left[\sigma\left(x_{*}+\delta_{k} f, y_{*}+\delta_{k} d\right)-\sigma\left(x_{*}, y_{*}\right)\right] \delta_{k}^{-1} \\
&= \lim _{\delta \rightarrow+0}\left[\sigma\left(x_{*}+\delta f, y_{*}+\delta d\right)-\sigma\left(x_{*}, y_{*}\right)\right] \delta^{-1} \\
&= \frac{\partial \sigma\left(x_{*}, y_{*}\right)}{\partial(f, d)} .
\end{aligned}
$$


Thus $\frac{\partial \sigma\left(x_{*}, y_{*}\right)}{\partial(f, p)} \leq \frac{\partial \sigma\left(x_{*}, y_{*}\right)}{\partial(f, d)}$ for any $d \in D a\left(x_{*}, y_{*}\right) \mid(f)$. It follows from here that $\inf _{d \in D a\left(x_{*}, y_{*}\right) \mid(f)} \frac{\partial \sigma\left(x_{*}, y_{*}\right)}{\partial(f, d)} \geq \frac{\partial \sigma\left(x_{*}, y_{*}\right)}{\partial(f, p)}$.

Since

$$
D^{*} Y_{*}\left(x_{*}, y_{*}\right)\left|(f) \subset D Y_{*}\left(x_{*}, y_{*}\right)\right|(f) \subset D a\left(x_{*}, y_{*}\right) \mid(f),
$$

then for any $p \in D^{*} Y_{*}\left(x_{*}, y_{*}\right)\left|(f), p \in D a\left(x_{*}, y_{*}\right)\right|(f)$ and it follows that the relation

$$
\inf _{d \in D a\left(x_{*}, y_{*}\right) \mid(f)} \frac{\partial \sigma\left(x_{*}, y_{*}\right)}{\partial(f, d)} \leq \frac{\partial \sigma\left(x_{*}, y_{*}\right)}{\partial(f, p)}
$$

is satisfied. In that case, it follows from the above two inequalities that the statement holds.

Theorem 4. Let the set-valued map $Y_{*}(\cdot): \mathbb{R}^{n} \rightarrow \operatorname{comp}\left(\mathbb{R}^{m}\right)$ be in the form (3.2). Suppose that there exists a $y_{*} \in Y_{*}\left(x_{*}\right)$ such that $\sigma(\cdot, \cdot)$ is a derivable function at the point $\left(x_{*}, y_{*}\right)$ in the direction $(f, d)$ for all $d \in \mathbb{R}^{m}$. Then

$$
D Y_{*}\left(x_{*}, y_{*}\right) \mid(f) \subset\left\{p \in \mathbb{R}^{m}: \inf _{d \in D^{*} a\left(x_{*}, y_{*}\right) \mid(f)} \frac{\partial \sigma\left(x_{*}, y_{*}\right)}{\partial(f, d)} \geq \frac{\partial \sigma\left(x_{*}, y_{*}\right)}{\partial(f, p)}\right\} .
$$

Proof. Let $y_{*} \in Y_{*}\left(x_{*}\right)$ such that $\sigma(\cdot, \cdot)$ is a derivable function at the point $\left(x_{*}, y_{*}\right)$ in direction $(f, d)$ for all $d \in \mathbb{R}^{m}$.

Let $D Y_{*}\left(x_{*}, y_{*}\right) \mid(f)=\varnothing$. Then statement (3.5) holds.

Let $D^{*} a\left(x_{*}, y_{*}\right) \mid(f)=\varnothing$. Then

$$
+\infty=\inf _{d \in D^{*} a\left(x_{*}, y_{*}\right) \mid(f)} \frac{\partial \sigma\left(x_{*}, y_{*}\right)}{\partial(f, d)}>\frac{\partial \sigma\left(x_{*}, y_{*}\right)}{\partial(f, p)}
$$

and it follows that the statement (3.5) holds.

Now let $D Y_{*}\left(x_{*}, y_{*}\right) \mid(f) \neq \varnothing$ and $D^{*} a\left(x_{*}, y_{*}\right) \mid(f) \neq \varnothing$. Let us take $p \in$ $D Y_{*}\left(x_{*}, y_{*}\right) \mid(f)$. Then from the definition of $D Y_{*}\left(x_{*}, y_{*}\right) \mid(f)$, there exists a sequence $y_{k} \in Y_{*}\left(x_{*}+\delta_{k} f\right)$, where $\delta_{k}>0$ and $\delta_{k} \rightarrow+0$ as $k \rightarrow \infty$ such that

$$
y_{k}=y_{*}+\delta_{k} p+o_{1}\left(\delta_{k}\right)
$$

where $\left\|o_{1}\left(\delta_{k}\right)\right\| / \delta_{k} \rightarrow 0$ as $k \rightarrow \infty$. Since

$$
Y_{*}(x)=\left\{y_{*} \in a(x): \sigma(x, y) \geq \sigma\left(x, y_{*}\right), \forall y \in a(x)\right\},
$$

then it follows that for any $y \in a\left(x_{*}+\delta_{k} f\right)$,

$$
\sigma\left(x_{*}+\delta_{k} f, y\right) \geq \sigma\left(x_{*}+\delta_{k} f, y_{*}+\delta_{k} p+o_{1}\left(\delta_{k}\right)\right) .
$$

Choose any $d \in D^{*} a\left(x_{*}, y_{*}\right) \mid(f)$. Then from the definition of $D^{*} a\left(x_{*}, y_{*}\right) \mid(f)$, there exists a $\delta_{*}>0$ such that for all $\delta \in\left[0, \delta_{*}\right]$,

$$
y(\delta)=y_{*}+\delta d+o_{2}(\delta) \in a\left(x_{*}+\delta f\right)
$$


where $\left\|o_{2}(\delta)\right\| \delta^{-1} \rightarrow 0$ as $\delta \rightarrow+0$. In that case from (3.6) since $\delta_{k} \rightarrow+0$, then it follows that there exists a $k_{0} \in \mathbb{N}$ such that $\delta_{k} \in\left[0, \delta_{*}\right]$ for all $k \geq k_{0}$ and

$$
\begin{aligned}
\sigma\left(x_{*}+\delta_{k} f, y_{*}+\delta_{k} d+o_{2}\left(\delta_{k}\right)\right) & -\sigma\left(x_{*}, y_{*}\right) \\
& \geq \sigma\left(x_{*}+\delta_{k} f, y_{*}+\delta_{k} p+o_{1}\left(\delta_{k}\right)\right)-\sigma\left(x_{*}, y_{*}\right) .
\end{aligned}
$$

Since the function $\sigma(\cdot, \cdot)$ is locally Lipschitz on $\mathbb{R}^{m}$, then it follows that for $k=1$, $2, \ldots$, there exist $L_{1}>0$ and $L_{2}>0$ such that

$$
\begin{aligned}
\mid \sigma\left(x_{*}+\delta_{k} f, y_{*}+\delta_{k} p+o_{1}\left(\delta_{k}\right)-\sigma\left(x_{*}+\delta_{k} f, y_{*}+\delta_{k} p\right) \mid\right. & \leq L_{1}\left\|o_{1}\left(\delta_{k}\right)\right\|, \\
\left|\sigma\left(x_{*}+\delta_{k} f, y_{*}+\delta_{k} d+o_{2}\left(\delta_{k}\right)\right)-\sigma\left(x_{*}+\delta_{k} f, y_{*}+\delta_{k} d\right)\right| & \leq L_{2}\left\|o_{2}\left(\delta_{k}\right)\right\| .
\end{aligned}
$$

Consequently, we have

$$
\begin{aligned}
\frac{\partial \sigma\left(x_{*}, y_{*}\right)}{\partial(f, p)}= & \lim _{\delta \rightarrow+0}\left[\sigma\left(x_{*}+\delta f, y_{*}+\delta p\right)-\sigma\left(x_{*}, y_{*}\right)\right] \delta^{-1} \\
= & \lim _{k \rightarrow \infty}\left[\sigma\left(x_{*}+\delta_{k} f, y_{*}+\delta_{k} p\right)-\sigma\left(x_{*}, y_{*}\right)\right] \delta_{k}^{-1} \\
= & \lim _{k \rightarrow \infty}\left[\sigma\left(x_{*}+\delta_{k} f, y_{*}+\delta_{k} p\right)-\sigma\left(x_{*}+\delta_{k} f, y_{*}+\delta_{k} p+o_{1}\left(\delta_{k}\right)\right)\right. \\
& \left.+\sigma\left(x_{*}+\delta_{k} f, y_{*}+\delta_{k} p+o_{1}\left(\delta_{k}\right)\right)-\sigma\left(x_{*}, y_{*}\right)\right] \delta_{k}^{-1} \\
\leq & \lim _{k \rightarrow \infty}\left[L_{1}\left\|o_{1}\left(\delta_{k}\right)\right\|+\sigma\left(x_{*}+\delta_{k} f, y_{*}+\delta_{k} p+o_{1}\left(\delta_{k}\right)\right)-\sigma\left(x_{*}, y_{*}\right)\right] \delta_{k}^{-1} \\
= & \lim _{k \rightarrow \infty}\left[\sigma\left(x_{*}+\delta_{k} f, y_{*}+\delta_{k} p+o_{1}\left(\delta_{k}\right)\right)-\sigma\left(x_{*}, y_{*}\right)\right] \delta_{k}^{-1} \\
\leq & \lim _{k \rightarrow \infty}\left[\sigma\left(x_{*}+\delta_{k} f, y_{*}+\delta_{k} d+o_{2}\left(\delta_{k}\right)\right)-\sigma\left(x_{*}, y_{*}\right)\right] \delta_{k}^{-1} \\
= & \lim _{k \rightarrow \infty}\left[\sigma\left(x_{*}+\delta_{k} f, y_{*}+\delta_{k} d+o_{2}\left(\delta_{k}\right)\right)-\sigma\left(x_{*}+\delta_{k} f, y_{*}+\delta_{k} d\right)\right. \\
& \left.+\sigma\left(x_{*}+\delta_{k} f, y_{*}+\delta_{k} d\right)-\sigma\left(x_{*}, y_{*}\right)\right] \delta_{k}^{-1} \\
\leq & \lim _{k \rightarrow \infty} L_{2}\left\|o_{2}\left(\delta_{k}\right)\right\| \delta_{k}^{-1}+\lim _{k \rightarrow \infty}\left[\sigma\left(x_{*}+\delta_{k} f, y_{*}+\delta_{k} d\right)-\sigma\left(x_{*}, y_{*}\right)\right] \delta_{k}^{-1} \\
= & \lim _{k \rightarrow \infty}\left[\sigma\left(x_{*}+\delta_{k} f, y_{*}+\delta_{k} d\right)-\sigma\left(x_{*}, y_{*}\right)\right] \delta_{k}^{-1} \\
= & \lim _{\delta \rightarrow+0}\left[\sigma\left(x_{*}+\delta f, y_{*}+\delta d\right)-\sigma\left(x_{*}, y_{*}\right)\right] \delta^{-1} \\
= & \frac{\partial \sigma\left(x_{*}, y_{*}\right)}{\partial(f, d)} .
\end{aligned}
$$

Thus, $\frac{\partial \sigma\left(x_{*}, y_{*}\right)}{\partial(f, p)} \leq \frac{\partial \sigma\left(x_{*}, y_{*}\right)}{\partial(f, d)}$ for any $d \in D^{*} a\left(x_{*}, y_{*}\right) \mid(f)$. It follows from here that

$$
\inf _{d \in D^{*} a\left(x_{*}, y_{*}\right) \mid(f)} \frac{\partial \sigma\left(x_{*}, y_{*}\right)}{\partial(f, d)} \geq \frac{\partial \sigma\left(x_{*}, y_{*}\right)}{\partial(f, p)}
$$

and hence the statement holds. 
Corollary 1. Let the set-valued map $Y_{*}(\cdot): \mathbb{R}^{n} \rightarrow \operatorname{comp}\left(\mathbb{R}^{m}\right)$ be in the form (3.2). Suppose that there exists a $y_{*} \in Y_{*}\left(x_{*}\right)$ such that $\sigma(\cdot, \cdot)$ is a differentiable function at the point $\left(x_{*}, y_{*}\right)$. Then

$$
D^{*} Y_{*}\left(x_{*}, y_{*}\right) \mid(f) \subset\left\{p \in \mathbb{R}^{m}: \inf _{d \in D a\left(x_{*}, y_{*}\right) \mid(f)}\left\langle\frac{\partial \sigma\left(x_{*}, y_{*}\right)}{\partial y}, d\right\rangle=\left\langle\frac{\partial \sigma\left(x_{*}, y_{*}\right)}{\partial y}, p\right\rangle\right\},
$$

where the symbol $\langle\cdot, \cdot\rangle$ denotes the inner product.

Proof. Since $\sigma(\cdot, \cdot)$ is a differentiable function at the point $\left(x_{*}, y_{*}\right)$, then it is a derivable function at the point $\left(x_{*}, y_{*}\right)$ in any direction $(f, d) \in \mathbb{R}^{n} \times \mathbb{R}^{m}$ and

$$
\frac{\partial \sigma\left(x_{*}, y_{*}\right)}{\partial(f, d)}=\left\langle\frac{\partial \sigma\left(x_{*}, y_{*}\right)}{\partial x}, f\right\rangle=\left\langle\frac{\partial \sigma\left(x_{*}, y_{*}\right)}{\partial y}, d\right\rangle .
$$

Then from Theorem 3, we obtain the corollary.

Corollary 2. Let the set-valued map $Y_{*}(\cdot): \mathbb{R}^{n} \rightarrow \operatorname{comp}\left(\mathbb{R}^{m}\right)$ be in the form (3.2). Suppose that there exists a $y_{*} \in Y_{*}\left(x_{*}\right)$ such that $\sigma(\cdot, \cdot)$ is a differentiable function at the point $\left(x_{*}, y_{*}\right)$. Then

$$
D Y_{*}\left(x_{*}, y_{*}\right) \mid(f) \subset\left\{p \in \mathbb{R}^{m}: \inf _{d \in D^{*} a\left(x_{*}, y_{*}\right) \mid(f)}\left\langle\frac{\partial \sigma\left(x_{*}, y_{*}\right)}{\partial y}, d\right\rangle \geq\left\langle\frac{\partial \sigma\left(x_{*}, y_{*}\right)}{\partial y}, p\right)\right\},
$$

where the symbol $\langle\cdot, \cdot\rangle$ denotes the inner product.

Proof. It is similar to that of Theorem 4.

Now, we give an example for the above theorems.

Example 1. Take a constant vector $l \in \mathbb{R}^{m} \cdot \sigma(\cdot, \cdot): \mathbb{R}^{m} \times \mathbb{R}^{m} \rightarrow \mathbb{R}$ is defined by

$$
(x, y) \rightarrow \sigma(x, y)=\langle l, y\rangle
$$

and $a(\cdot)$ is defined by

$$
x \rightarrow a(x)=\left\{y \in \mathbb{R}^{m}: b(x, y) \geq 0\right\}
$$

where $b(\cdot, \cdot): \mathbb{R}^{m} \times \mathbb{R}^{m} \rightarrow \mathbb{R}$ is continuous differentiable such that $a(x)$ is bounded for all $x \in \mathbb{R}^{m}$ and $\frac{\partial b(x, y)}{\partial y} \neq 0$ for any $y \in L(x)$ where

$$
L(x)=\left\{y \in \mathbb{R}^{m}: b(x, y)=0\right\} .
$$

Then $a(x): \mathbb{R}^{m} \rightarrow \operatorname{comp}\left(\mathbb{R}^{m}\right)$ and

$$
Y_{*}(x) \subset \partial a(x) \subset L(x)
$$

where $\partial a(x)$ is a boundary of $a(x)$. It is shown that

$$
Y_{*}(x) \subset K(x)
$$

where

$$
K(x)=\left\{y_{0} \in a(x):\langle l, d\rangle \geq 0 \text { for all } d \in T_{a(x)}\left(y_{0}\right)\right\},
$$

and 


$$
\begin{aligned}
T_{a(x)}\left(y_{0}\right)=\left\{d \in \mathbb{R}^{m}: \exists \delta_{k}>0\left(\delta_{k} \rightarrow+0\right.\right. & \text { as } k \rightarrow \infty), \\
& \left.\quad \exists y_{k} \in a(x) \ni d=\lim _{k \rightarrow \infty} \frac{y_{k}-y_{0}}{\delta_{k}}\right\},
\end{aligned}
$$

where $T_{a(x)}\left(y_{0}\right)$ is an upper contingent cone of $a(x)$ at $y_{0}$.

Let $y_{*} \in Y_{*}(x)$. Then $y_{*} \in \partial a(x)$ and $b\left(x, y_{*}\right)=0$. Since $\frac{\partial b\left(x, y_{*}\right)}{\partial y} \neq 0$, we get

$$
T_{a(x)}\left(y_{0}\right)=\left\{d \in \mathbb{R}^{m}:\left\langle\frac{\partial b\left(x, y_{*}\right)}{\partial y}, d\right\rangle \geq 0\right\}
$$

and from [7, Corollary 2],

$$
\begin{aligned}
D a\left(x, y_{*}\right) \mid(f) & =D^{*} a\left(x, y_{*}\right) \mid(f) \\
& =\left\{d \in \mathbb{R}^{m}:\left\langle\frac{\partial b\left(x, y_{*}\right)}{\partial x}, f\right\rangle+\left\langle\frac{\partial b\left(x, y_{*}\right)}{\partial y}, d\right\rangle \geq 0\right\} .
\end{aligned}
$$

Since $y_{*} \in Y_{*}(x)$, then it follows from (3.7) that $y_{*} \in K(x)$ and since $y_{*} \in K(x)$, then from (3.8), $\langle l, d\rangle \geq 0$ for all $d \in T_{a(x)}\left(y_{*}\right)$. Then from (3.9) we get

$$
\langle l, d\rangle \geq 0 \text { for all } d \in \mathbb{R}^{m} \text { such that }\left\langle\frac{\partial b\left(x, y_{*}\right)}{\partial y}, d\right\rangle \geq 0 .
$$

This yields $l=\alpha(x) \frac{\partial b\left(x, y_{*}\right)}{\partial y}$ with $\alpha(x)>0$, and follows that

$$
Y_{*}(x) \subset\left\{y_{*} \in \partial a(x): l=\alpha(x) \frac{\partial b\left(x, y_{*}\right)}{\partial y}, \alpha(x)>0\right\} .
$$

Therefore, Theorems 3 and 4 and statements (3.10), (3.11) yield

$$
\begin{aligned}
D^{*} Y_{*}\left(x, y_{*}\right) \mid(f) & \subset\left\{p \in \mathbb{R}^{m}: \min _{d \in D a\left(x, y_{*}\right) \mid(f)}\langle l, d\rangle=\langle l, p\rangle\right\} \\
& =\left\{p \in \mathbb{R}^{m}:\langle l, p\rangle=-\alpha(x)\left\langle\frac{\partial b\left(x, y_{*}\right)}{\partial x}, f\right\rangle\right\}, \\
D Y_{*}\left(x, y_{*}\right) \mid(f) & \subset\left\{p \in \mathbb{R}^{m}: \min _{d \in D^{*} a\left(x, y_{*}\right) \mid(f)}\langle l, d\rangle \geq\langle l, p\rangle\right\} \\
& =\left\{p \in \mathbb{R}^{m}:\langle l, p\rangle \leq-\alpha(x)\left\langle\frac{\partial b\left(x, y_{*}\right)}{\partial x}, f\right\rangle\right\} .
\end{aligned}
$$

\section{DiRECTIONAL DiFFERENTIABILITY OF MAX-MIN FUNCTION}

In [7], the directional lower and upper derivatives of max-min function are investigated by using the directional lower and upper derivative sets of max-min set-valued map. 
Now, in this paper, a sufficient condition ensuring the existence of the directional derivative of the max-min function is obtained by using the lower differentiability of max-min set-valued maps. Hence by using the lower different, a characterization of the upper and lower directional derivatives of max-min functions is obtained. For $A \subset \mathbb{R}^{n}$ and $C \subset \mathbb{R}^{n}$, we put $\beta(A, C)=\sup _{a \in A} d(a, C), d_{H}(A, C)=$ $\max \{\beta(A, C), \beta(C, A)\}$. It is known that $\left(\operatorname{comp}\left(\mathbb{R}^{n}\right), d_{H}(\cdot, \cdot)\right)$ is a metric space.

Definition 1. The set-valued map $a(\cdot): \mathbb{R}^{n} \rightarrow \operatorname{comp}\left(\mathbb{R}^{m}\right)$ is said to be lower differentiable at the point $x \in \mathbb{R}^{n}$ in direction $f \in \mathbb{R}^{n}$, if there exist $G^{-}(x, f) \in \operatorname{comp}\left(\mathbb{R}^{m}\right)$, $G^{+}(x, f) \in \operatorname{comp}\left(\mathbb{R}^{m}\right)$ such that

$$
\lim _{\delta \rightarrow+0} \frac{1}{\delta} \beta\left(a(x)+\delta G^{+}(x, f), a(x+\delta f)+\delta G^{-}(x, f)\right)=0 .
$$

In that case the pair $\left(G^{-}(x, f), G^{+}(x, f)\right)$ is said to be lower differential of the setvalued map $a(\cdot): \mathbb{R}^{n} \rightarrow \operatorname{comp}\left(\mathbb{R}^{m}\right)$ at the point $x \in \mathbb{R}^{n}$ in the direction $f \in \mathbb{R}^{n}$ [9].

In [9], the following two propositions are given.

Proposition 1. Let the set-valued map $a(\cdot): \mathbb{R}^{n} \rightarrow \operatorname{comp}\left(\mathbb{R}^{m}\right)$ be lower differentiable at the point $x \in \mathbb{R}^{n}$ in direction $f \in \mathbb{R}^{n}$. Then $D a(x, y) \mid(f) \neq \varnothing$ for every $y \in a(x)$.

Proposition 2. Let the set-valued map $a(\cdot): \mathbb{R}^{n} \rightarrow \operatorname{comp}\left(\mathbb{R}^{m}\right)$ be lower differentiable at the point $x \in \mathbb{R}^{n}$ in direction $f \in \mathbb{R}^{n}$ and the pair $\left(0, G^{+}(x, f)\right)$ be its lower differential. Then $D^{*} a(x, y) \mid(f) \neq \varnothing$ for every $y \in a(x)$.

In [7], the following two propositions characterizing upper and lower directional derivatives of max-min function $m(\cdot)$ are proved.

Proposition 3. For all $x \in \mathbb{R}^{n}$ and $f \in \mathbb{R}^{n}$

$$
\begin{aligned}
& \frac{\partial^{-} m(x)}{\partial f} \leq \inf _{(y, z) \in Y_{*}(x)} \inf _{(d, n) \in D Y_{*}(x, y, z) \mid(f)} \frac{\partial^{+} \sigma(x, y, z)}{\partial(f, d, n)}, \\
& \frac{\partial^{+} m(x)}{\partial f} \leq \inf _{(y, z) \in Y_{*}(x)} \inf _{(d, n) \in D^{*} Y_{*}(x, y, z) \mid(f)} \frac{\partial^{+} \sigma(x, y, z)}{\partial(f, d, n)} .
\end{aligned}
$$

Proposition 4. Let $x \in \mathbb{R}^{n}, f \in \mathbb{R}^{n}$ and there exists $\left(y_{*}, z_{*}\right) \in Y_{*}(x)$ such that $D Y_{*}\left(x, y_{*}, z_{*}\right) \mid(f) \neq \varnothing$. Then

$$
\frac{\partial^{+} m(x)}{\partial f} \geq \inf _{(y, z) \in Y_{*}(x)} \inf _{(d, n) \in D Y_{*}(x, y, z) \mid(f)} \frac{\partial^{-} \sigma(x, y, z)}{\partial(f, d, n)} .
$$

Moreover if there exists $\left(y^{*}, z^{*}\right) \in Y_{*}(x)$ such that $D^{*} Y_{*}\left(x, y^{*}, z^{*}\right) \mid(f) \neq \varnothing$ then

$$
\frac{\partial^{-} m(x)}{\partial f} \geq \inf _{(y, z) \in Y_{*}(x)} \inf _{(d, n) \in D^{*} Y_{*}(x, y, z) \mid(f)} \frac{\partial^{-} \sigma(x, y, z)}{\partial(f, d, n)} .
$$


Now, by using the lower differentiability of max-min set-valued maps, we give a characterization of the upper and lower directional derivatives of $m(\cdot)$ which follows from Propositions $1-4$.

Theorem 5. Suppose that the set-valued map $Y_{*}(\cdot): \mathbb{R}^{n} \rightarrow \operatorname{comp}\left(\mathbb{R}^{m} \times \mathbb{R}^{k}\right)$ is lower differentiable at the point $x \in \mathbb{R}^{n}$ in the direction $f \in \mathbb{R}^{n}$. Then

$$
\begin{aligned}
& \frac{\partial^{+} m(x)}{\partial f} \leq \inf _{(y, z) \in Y_{*}(x)} \inf _{(d, n) \in D^{*} Y_{*}(x, y, z) \mid(f)} \frac{\partial^{+} \sigma(x, y, z)}{\partial(f, d, n)}, \\
& \frac{\partial^{+} m(x)}{\partial f} \geq \inf _{(y, z) \in Y_{*}(x)} \inf _{(d, n) \in D Y_{*}(x, y, z) \mid(f)} \frac{\partial^{-} \sigma(x, y, z)}{\partial(f, d, n)} .
\end{aligned}
$$

Theorem 6. Suppose that the set-valued map $Y_{*}(\cdot): \mathbb{R}^{n} \rightarrow \operatorname{comp}\left(\mathbb{R}^{m} \times \mathbb{R}^{k}\right)$ is lower differentiable at the point $x \in \mathbb{R}^{n}$ in direction $f \in \mathbb{R}^{n}$ and $\left(0, G^{+}(x, f)\right)$ is its lower differential. Let the function $\sigma(\cdot, \cdot, \cdot): \mathbb{R}^{n} \times \mathbb{R}^{m} \times \mathbb{R}^{k} \rightarrow \mathbb{R}$ be directional differentiable at the point $(x, y, z)$ in direction $(f, d, n) \in \mathbb{R}^{n} \times \mathbb{R}^{m} \times \mathbb{R}^{k}$ for any $(y, z) \in Y_{*}(x)$ and $(d, n) \in \mathbb{R}^{m} \times \mathbb{R}^{k}$. Then

$$
\frac{\partial m(x)}{\partial f}=\inf _{(y, z) \in Y_{*}(x)} \inf _{(d, n) \in D Y_{*}(x, y, z) \mid(f)} \frac{\partial \sigma(x, y, z)}{\partial(f, d, n)} .
$$

\section{CONCLUSIONS}

Necessary statements are given for a minimum point and a maximum point of the max-min function. The estimations for the directional lower and upper derivative sets of max-min set-valued map which are used to state a characterization of the directional derivative of max-min function are given. Moreover, by using the lower differentiability of max-min set-valued maps, sufficient condition ensuring the existence of the directional derivative of the max-min function is obtained.

\section{REFERENCES}

[1] J.-P. Aubin and H. Frankowska, Set-valued analysis, ser. Systems \& Control: Foundations \& Applications. Boston, MA: Birkhäuser Boston Inc., 1990, vol. 2.

[2] F. H. Clarke, Y. S. Ledyaev, R. J. Stern, and P. R. Wolenski, "Qualitative properties of trajectories of control systems: a survey," J. Dynam. Control Systems, vol. 1, no. 1, pp. 1-48, 1995.

[3] F. H. Clarke and R. B. Vinter, "The relationship between the maximum principle and dynamic programming," SIAM J. Control Optim., vol. 25, no. 5, pp. 1291-1311, 1987.

[4] J. M. Danskin, The theory of max-min and its application to weapons allocation problems, ser. Econometrics and Operations Research, Vol. V. Springer-Verlag New York, Inc., New York, 1967.

[5] V. F. Demyanov and A. M. Rubinov, Constructive nonsmooth analysis, ser. Approximation \& Optimization. Frankfurt am Main: Peter Lang, 1995, vol. 7.

[6] V. F. Dem'yanov and L. V. Vasil'ev, Nondifferentiable optimization, ser. Translation Series in Mathematics and Engineering. New York: Optimization Software Inc. Publications Division, 1985, translated from the Russian by Tetsushi Sasagawa. 
[7] E. Ekici, "On the directional differentiability properties of the max-min function," Bol. Asoc. Mat. Venez., vol. 10, no. 1, pp. 35-42, 2003.

[8] H. G. Guseǐnov, A. I. Subbotin, and V. N. Ushakov, "Derivatives for multivalued mappings with applications to game-theoretical problems of control," Problems Control Inform. Theory/Problemy Upravlen. Teor. Inform., vol. 14, no. 3, pp. 155-167, R1-R14, 1985, with a Russian translation.

[9] K. G. Guseinov, Y. Kucuk, and E. Ekici, "Lower and upper directional differentiability of the set valued maps and their applications," Dyn. Contin. Discrete Impuls. Syst. Ser. A Math. Anal., vol. 10, no. 1-3, pp. 25-32, 2003, second International Conference on Dynamics of Continuous, Discrete and Impulsive Systems (London, ON, 2001).

[10] N. N. Krasovskiı̌ and A. I. Subbotin, Game-theoretical control problems, ser. Springer Series in Soviet Mathematics. New York: Springer-Verlag, 1988, translated from the Russian by Samuel Kotz.

[11] L. Minchenko and A. Volosevich, "Aspects of differentiability of multifunctions," in Proceedings of the 11th IFAC International Workshop Control Applications of Optimization, 3-6 July, St. Petersburg, Russia, vol. 1, 2000, pp. 239-243.

[12] E. S. Polovinkin and G. V. Smirnov, "Differentiation of multivalued mappings and properties of the solutions of differential inclusions," Dokl. Akad. Nauk SSSR, vol. 288, no. 2, pp. 296-301, 1986.

[13] N. N. Subbotina, "The maximum principle and the superdifferential of the value function," Problems Control Inform. Theory/Problemy Upravlen. Teor. Inform., vol. 18, no. 3, pp. 151-160, P1P10, 1989, with the Russian original.

[14] L. Thibault, "On subdifferentials of optimal value functions," SIAM J. Control Optim., vol. 29, no. 5, pp. 1019-1036, 1991 .

\section{Author's address}

\section{Erdal Ekici}

Department of Mathematics, Canakkale Onsekiz Mart University, Terzioglu Campus, 17020 Canakkale, Turkey

E-mail address: eekici@comu.edu.tr 